\title{
Advanced modulation formats for transmission systems
}

Tokle, Torger; Serbay, M.; Jensen, Jesper Bevensee; Rosenkranz, W.; Jeppesen, Palle

Published in:

Proceedings of The Optical Fiber Communication Conference and Exposition (OFC)

Link to article, DOI:

10.1109/OFC.2008.4528340

Publication date:

2008

Document Version

Publisher's PDF, also known as Version of record

Link back to DTU Orbit

Citation (APA):

Tokle, T., Serbay, M., Jensen, J. B., Rosenkranz, W., \& Jeppesen, P. (2008). Advanced modulation formats for transmission systems. Proceedings of The Optical Fiber Communication Conference and Exposition (OFC), OMl1. https://doi.org/10.1109/OFC.2008.4528340

\section{General rights}

Copyright and moral rights for the publications made accessible in the public portal are retained by the authors and/or other copyright owners and it is a condition of accessing publications that users recognise and abide by the legal requirements associated with these rights.

- Users may download and print one copy of any publication from the public portal for the purpose of private study or research.

- You may not further distribute the material or use it for any profit-making activity or commercial gain

- You may freely distribute the URL identifying the publication in the public portal 


\title{
Advanced Modulation Formats for Transmission Systems
}

\author{
Torger Tokle $^{1^{*}}$, Murat Serbay ${ }^{2}$, Jesper Bevensee Jensen ${ }^{1}$, Werner Rosenkranz ${ }^{2}$ and Palle Jeppesen ${ }^{1}$ \\ 1) COM•DTU, Technical University of Denmark, Building 345v, DK-2800 Kgs. Lyngby, Denmark. \\ *) Currently with OFS Fitel Denmark, Priorparken 680, 2605 Brøndby, Denmark. Email: ttokle@ ofsoptics.com. \\ 2) Chair for Communications, University of Kiel, Kaiserstraße 2, 24143 Kiel, Germany
}

\begin{abstract}
DQPSK has shown that multilevel modulation formats can enable new possibilities in optical communication systems. Here, we present several options to go to even more advanced formats using direct detection.

(C)2007 Optical Society of America

OCIS codes: (060.2330) Fiber optics communications; (060.4080) Modulation
\end{abstract}

\section{Introduction}

Historically, optical communication systems were using a very simple modulation format, simply sending light to signal a " 1 " and not sending light to signal a " 0 ". This modulation format, known as on-off keying (OOK), is still the preferred modulation format for most links due its easy implementation [1]. However, as the transmission distances and the per channel bit rates increase, and the channel spacing decrease, more advanced modulation formats have been suggested to mitigate nonlinear transmission impairments, improve the receiver sensitivity or to facilitate a per channel bit rate increase beyond the limits of binary systems.

One promising advanced modulation format is the 4-ary differential quadrature phase shift keying (DQPSK) format. With DQPSK the data is encoded into one out of four different symbols, thus enabling simultaneous transmission of two bits per symbol. The main benefit of DQPSK is the reduced spectral width, which is about half that of a binary format at the same bit rate. By 2003, DQPSK systems were sufficiently mature to allow demonstrations with good performance at bit rates above $10 \mathrm{Gbit} / \mathrm{s}[2,3]$. This opened up a wave of experiments that have demonstrated several benefits of multilevel modulation formats. The increased spectral efficiency of these new formats has lead to a number of recent hero transmission experiments (see e.g. [4]).

In order to increase the spectral efficiency further, even more advanced modulation formats are required. In this paper, we present a number of experiments investigating how to go beyond DQPSK. Experiments using combinations of amplitude shift keying (ASK) and DQPSK, DQPSK with inverse-return-to-zero (RZ) pulse shape and differential 8-ary phase shift keying (D8PSK) are presented.

\section{Combination of phase and amplitude modulation - DQPSK-ASK}

By adding binary amplitude modulation (amplitude shift keying - ASK) to a DQPSK signal, the bit rate can be increased by $50 \%$. This is obtained by only small modifications to the transmitter and receiver of a standard DQPSK setup. In the transmitter an extra modulator is added to impose the amplitude modulation, and in the receiver an extra photodiode detects the amplitude information. For details on transmitter receiver designs, please refer to [5].

We upgraded an $80 \mathrm{Gbit} / \mathrm{s}$ RZ-DQPSK system at 40 Gbaud with ASK modulation, and obtained a $120 \mathrm{Gbit} / \mathrm{s}$ RZ-DQPSK-ASK signal. This signal is suitable for carrying $100 \mathrm{Gbit} / \mathrm{s}$ Ethernet including forward error correction (FEC) overhead. Thus we can generate a $100 \mathrm{Gbit} / \mathrm{s}$ Ethernet signal that is compatible with existing $40 \mathrm{Gbit} / \mathrm{s}$ WDM systems, and only using commercially available $40 \mathrm{Gbit} / \mathrm{s}$ equipment.

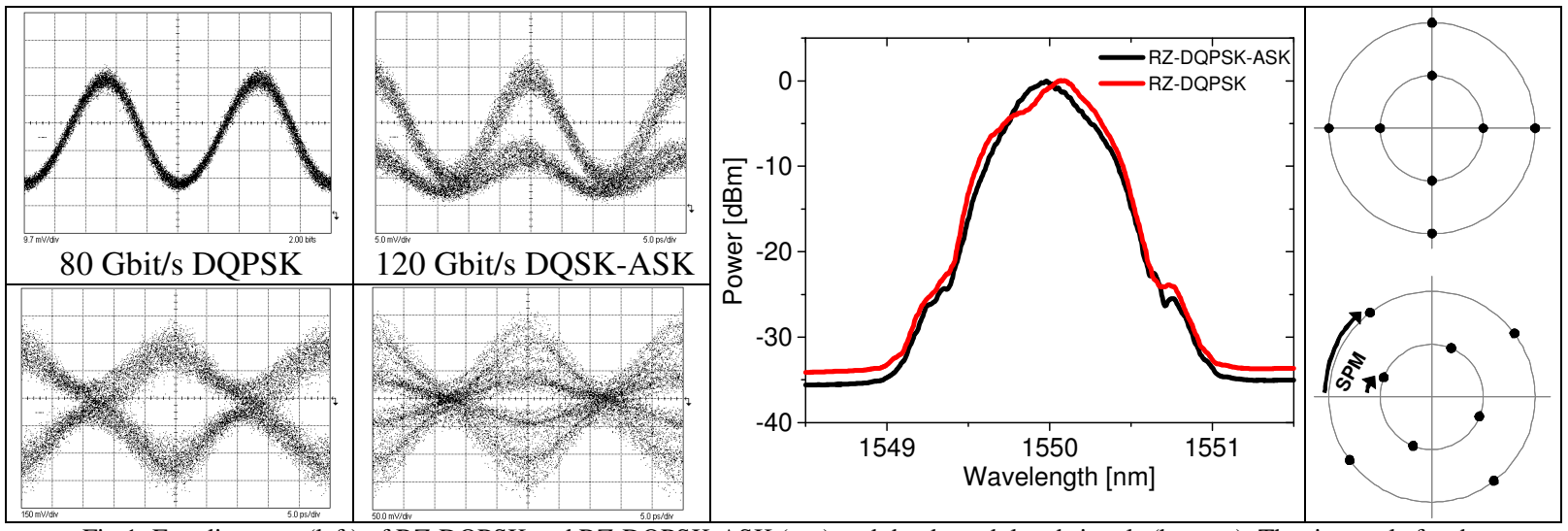

Fig 1: Eye diagrams (left) of RZ-DQPSK and RZ-DQPSK-ASK (top) and the demodulated signals (bottom). The timescale for the eye diagrams is 5 ps per division. The optical power spectrum (centre) shows that the two signals have the same bandwidth. At right, the symbol constellation diagrams for DQPSK-ASK before (top) and after (bottom) degradation by SPM. 


\section{OMI1.pdf}

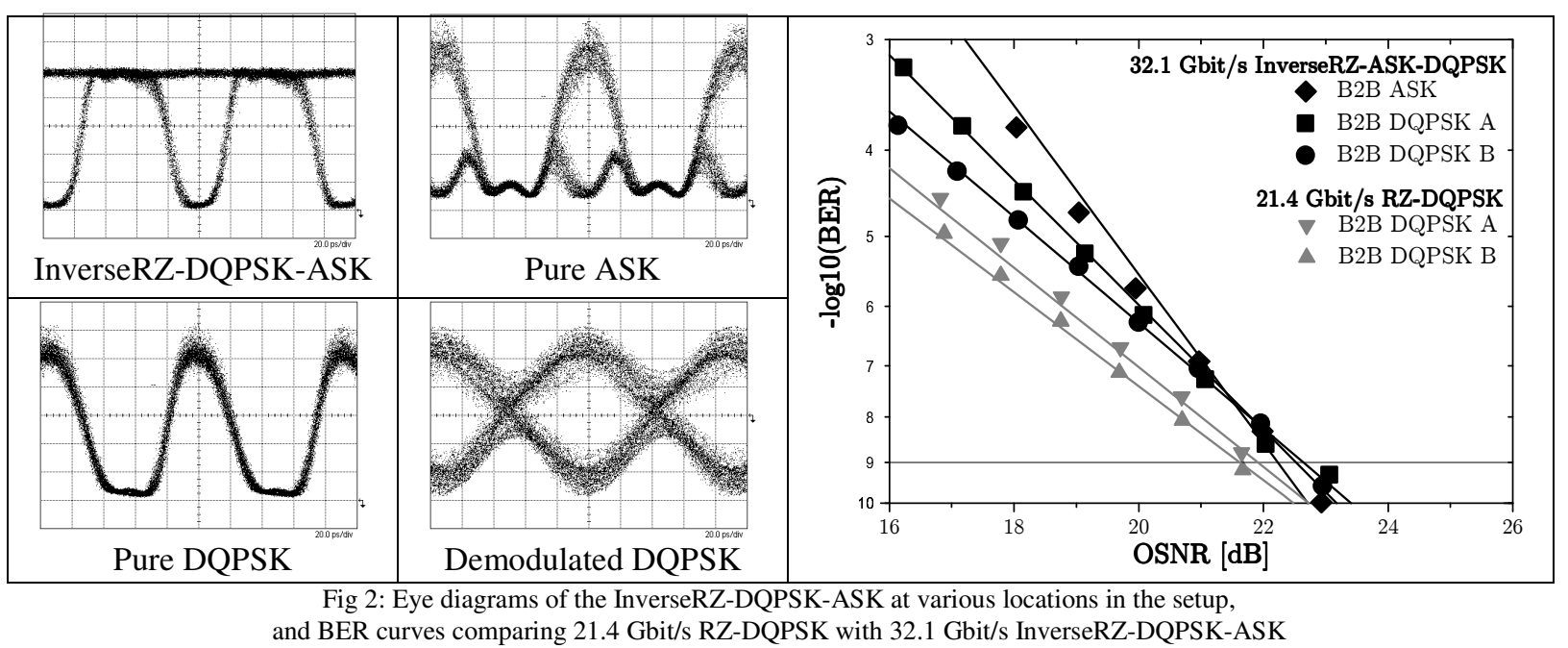

In order to illustrate the differences between DQPSK and DQPSK-ASK, we plot the eye diagrams in Fig. 1. The optical pulse width is the same, but since more information is stored in each pulse, the bit rate is $80 \mathrm{Gbit} / \mathrm{s}$ for DQPSK and $120 \mathrm{Gbit} / \mathrm{s}$ for DQPSK-ASK. Also shown in Fig. 1 is the optical power spectrum, and it is seen that the spectral width is not increased when going from DQPSK to DQPSK-ASK, despite the $50 \%$ bit rate increase.

When combining amplitude modulation and phase modulation, the amplitude extinction ratio is a trade-off between good eye opening for the amplitude signal and good eye opening for the demodulated phase signal. We found that an extinction ratio of $4.5 \mathrm{~dB}$ resulted in optimum performance for the system as a whole, significantly lower than the theoretical value due to amplitude jitter on the driving signals.

We successfully demonstrated the upgrade from $80 \mathrm{Gbit} / \mathrm{s}$ DQPSK to $120 \mathrm{Gbit} / \mathrm{s}$ ASK-DQPSK but noticed a $10 \mathrm{~dB}$ receiver sensitivity penalty at a bit error rate of $10^{-9}[5]$. This penalty is mainly due to the reduced DQPSK eye opening and the low extinction ratio of the amplitude modulation.

One drawback of combined phase and amplitude modulation is nonlinearities induced by self-phase modulation (SPM). Since the power of different symbols varies due to the amplitude modulation, the SPM-induced phase shift is not the same for all symbols, leading to distortions. This is illustrated in Fig. 1, where the symbol constellation of undistorted DQPSK-ASK is compared with a case where the signal has been distorted by SPM. As the "outer ring" has higher power, it will experience a higher SPM-induced phase change, and thus distort the signal. Although simple solutions have been proposed to overcome this [6], it can be a limiting factor for long-haul transmission using these modulation formats.

\section{Inverse RZ pulse carving}

One interesting method to allow for better integration of phase and amplitude modulation formats is to use inverseRZ pulse carving rather than normal RZ. This allows the separation of the phase and amplitude modulation in such a way that the phase modulation is stored in the part of the waveform that has high and constant power. Thanks to this, the amplitude modulation is allowed to use high (ideally infinite) extinction ratio at the same time as the phase modulation is hardly affected. InverseRZ can be modulated onto an optical carrier by a properly biased MachZehnder modulator driven by an electrical RZ signal [7], or using optical signal processing to invert a conventional RZ signal [8]. The electrical RZ signal can be generated by using the AND operation with the data signal and a clock signal as inputs.

At the receiver, one can use pulse carving to separate the amplitude and phase modulation. This is obtained by modulating a clock signal such that the resulting eye only contains the amplitude modulation for the ASK receiver and the phase modulation for the DQPSK receiver. Fig. 2 shows the eye diagrams of an InverseRZ signal out of the transmitter, after the receiver pulse carving and the DQPSK signal after demodulation.

In [7], we demonstrated that the upgrade from $21.4 \mathrm{Gbit} / \mathrm{s}$ RZ-DQPSK to $32.1 \mathrm{Gbit} / \mathrm{s}$ InverseRZ-DQPSK-ASK requires only $0.9 \mathrm{~dB}$ higher OSNR. This clearly demonstrates the benefits of using InverseRZ as a capacity upgrade path from DQPSK to more advanced modulation formats.

\section{Differential 8-ary phase shift keying}

Instead of adding amplitude modulation to a DQPSK signal, one can also add more phase levels. A DQPSK

transmitter requires little modifications to generate a D8PSK signal. By simply adding a phase modulator driven with a binary data signal after the DQPSK transmitter, a D8PSK signal with eight different phase changes between 


\section{OMI1.pdf}
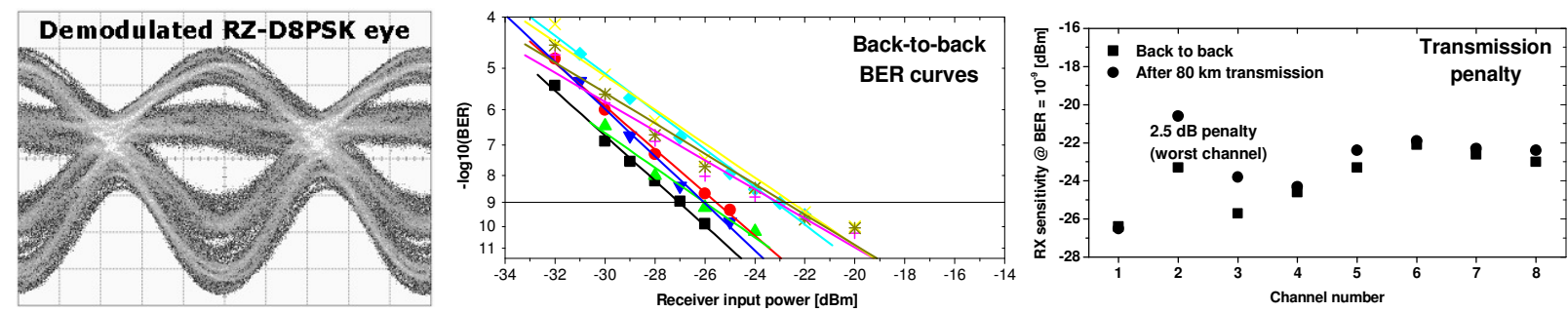

Fig 3: Eye diagram of the demodulated D8PSK signal (left), BER curves for all eight channels (centre) and receiver sensitivities before and after transmission (right)

consecutive symbols is generated $[9,10]$. The receiver, on the other hand, requires larger modification. Four sets of delay demodulators and balanced photodiodes plus an XOR gate are required ${ }^{1)}$. This is twice the complexity of a DQPSK receiver, a significant complexity increase for a 50\% bit rate increase. Despite the complex receiver, D8PSK is very interesting. It carries three bits per symbol while keeping the power envelope of each pulse constant, which is beneficial for the nonlinear performance.

In our study, we upgraded a $20 \mathrm{Gbit} / \mathrm{s}$ DQPSK system to $30 \mathrm{Gbit} / \mathrm{s}$ D8PSK, and verified the transmission performance in a wavelength division multiplexed (WDM) system. The setup is similar to that in [13]. Fig. 3 shows the eye diagram of the demodulated D8PSK signal, BER curves and power penalty curves after transmission over an $80 \mathrm{~km}$ standard single mode fibre (SSMF) link. Although the implementation penalty is high, we see that almost all WDM channels have very small transmission penalty. We attribute most of the differences between the channels to slightly different laser linewidths, and drifts in the setup during the measurement. This experiment shows that even such advanced modulation formats as D8PSK can show good transmission performance, even after transmission in a WDM system.

\section{Conclusion}

We have discussed several paths to go from direct detection DQPSK systems towards systems with more advanced modulation formats. With three bits per symbol at $40 \mathrm{Gbaud}$ symbol rate, the resulting $120 \mathrm{Gbit} / \mathrm{s}$ signal can carry $100 \mathrm{Gbit} / \mathrm{s}$ Ethernet traffic while having the same spectral width as a $40 \mathrm{Gbit} / \mathrm{s}$ signal. InverseRZ almost removes the implementation penalty and mitigates some of the sensitivity towards SPM-nonlinear degradation. D8PSK can be a suitable upgrade path from DQPSK when a constant symbol power shape is required.

Our experiments have shown that there are many promising technical solutions available for system designers to choose from when designing the next generation optical communication system. The optimum modulation format will as always depend on the actual system parameters, but it is clear that InverseRZ is a key technology to allow combination of phase and amplitude modulation in direct detection systems, reducing the implementation penalty from $10 \mathrm{~dB}$ to less than $1 \mathrm{~dB}$.

\section{References}

[1] N. S. Bergano, M. Nissov, A. Pilipetskii, J.-X. Cai, C. Davidson, and B. Bakhshi. Chirped return-to-zero formats for ultra long-haul fiber communications, IEEE/LEOS 2004 Workshop on Advanced Modulation Formats, Paper ThA1, July 2004.

[2] C. Wree, J. Leibrich, J. Eick, and W. Rosenkranz. Experimental investigation of receiver sensitivity of RZ-DQPSK modulation format using balanced detection, OFC'03, Paper ThE5, 2003.

[3] P. S. Cho, V. S. Grigoryan, Y. A. Godin, A. Salamon, and Y. Achiam. Transmission of 25 Gb/s RZ-DQPSK signals with 25 GHz channel spacing over $1000 \mathrm{~km}$ of SMF-28 fiber, Photonics Technology Letters, vol. 15, no. 3, pp. 473-475, March 2003.

[4] A. H. Gnauck, G. Charlet, P. Tran, P. J. Winzer, C. R. Doerr, J. C. Centanni, E. C. Burrows, T. Kawanishi, T. Kawanishi, T. Sakamoto, and K. Higuma. 25.6 Tbit/s C+E-band transmission of polarization- multiplexed RZ-DQPSK signal, OFC'07, Paper PDP19, March 2007.

[5] T. Tokle, M. Serbay, J. B. Jensen, Y. Geng, W. Rosenkranz, and P. Jeppesen. Investigation of multilevel phase and amplitude modulation formats in combination with polarisation multiplexing up to $240 \mathrm{Gbit} / \mathrm{s}$, Photonics Technology Letters, vol. 18, no. 20, pp. 2090-2092, October 2006.

[6] J. Hansryd, J. van Howe, and C. Xu. Nonlinear crosstalk and compensation in QDPASK optical communication systems, Photonics Technology Letters, vol. 16, no. 8, pp. 1975-1977, August 2004.

[7] T. Tokle, M. Serbay, W. Rosenkranz, and P. Jeppesen. 32.1 Gbit/s InverseRZ-ASK-DQPSK modulation with low implementation penalty, LEOS'06, Paper WH2, October 2006.

[8] T. Miyazaki and F. Kubota. DPSK over inverse-RZ for 2-bit per symbol transmission, OECC'04, Paper 14C3-2, July 2004.

[9] C. Kim and G. Li. Direct-detection optical differential 8-level phase-shift keying (OD8PSK) for spectrally efficient transmission, Optics Express, vol. 12 , no. 15 , pp. $3415-3421$, July 2004.

[10] M. Serbay, C. Wree, and W. Rosenkranz. Experimental investigation of RZ-8DPSK at 3×10.7 Gb/s, LEOS'05, pp. 483 - 484, October 2005.

[11] Y. Han, C. Kim, and G. Li. Simplified receiver implementation for optical differential 8-level phase-shift keying, Electronics Letters, vol. 40, no. 21, pp. 1372-1373, October 2004

[12] D. L. Hosung Yoon and N. Park. Performance comparison of optical 8-ary differential phase-shift keying systems with different electrical decision schemes, Optics Express, vol. 13, no. 2, pp. 371-376, January 2005.

[13] J. B. Jensen, T. Tokle, C. Peucheret, and P. Jeppesen. Transmission of multilevel 60 Gbit/s polarization multiplexed RZ-D8PSK using only 10 Gbit/s equipment, OFC'07, Paper OWM4, March 2007.

1) Optionally, one might use receivers with two delay demodulators and balanced photo diode pairs [11]. However, this requires analogue electrical processing on the received data and gives a worse receiver sensitivity [12]. 